\title{
Procesos inclusivos de la educación parvularia desde la mirada de agentes educativas
}

Inclusive processes of preschool education from the perspective of educational agents

\section{Volumen 21, Número 1 \\ Enero - Abril \\ pp. 1-28}

\author{
Fernanda Rubilar Bugueño \\ Daniela Guzmán Sanhueza
}

Citar este documento según modelo APA

Rubilar Bugueño, Fernanda. y Guzmán Sanhueza, Daniela. (2021). Procesos inclusivos de la educación parvularia desde la mirada de agentes educativas. Revista Actualidades Investigativas en Educación, 21(1), 1-28. Doi. 10.15517/aie.v21i1.42517 


\section{Procesos inclusivos de la educación parvularia desde la mirada de agentes educativas \\ Inclusive processes of preschool education from the perspective of educational agents}

\section{Fernanda Rubilar Bugueño ${ }^{1}$ Daniela Guzmán Sanhueza²}

Resumen: El artículo se lleva a cabo por la necesidad de visualizar los procesos inclusivos desde la mirada de asistentes, educadoras y directoras pertenecientes a Fundación Integra, Junta Nacional de Jardines Infantiles (JUNJI) y jardines administrados por la Municipalidad Vía Transferencia de Fondos (VTF) de la comuna de Copiapó de la Tercera región de Chile, instituciones que, por lineamientos se declaran inclusivas y reconocidas por trabajar con la población infantil más vulnerable de Chile. Esta investigación tuvo por objetivo principal analizar los procesos inclusivos de la educación parvularia en la comuna Copiapó. Utilizó el enfoque cualitativo con un paradigma interpretativo, mediante entrevistas semiestructuradas aplicadas a nueve agentes educativas en el año 2019 , abordando categorías: significados, metodologías de aprendizaje y obstaculizadores y facilitadores. Los resultados indican que, si bien las agentes educativas utilizan metodologías de aprendizajes que favorecen la participación de cada infante, existe un desconocimiento conceptual de educación inclusiva y discapacidad. Respecto a obstaculizadores se evidencia escasas capacitaciones en materia de discapacidad, insuficiente material didáctico, formación profesional desactualizada y poca accesibilidad infraestructural en los jardines. En cuanto a facilitadores se destacan redes externas, la gestión de matrícula y la relación de las agentes educativas con la familia. Se concluye que la incoherencia de conceptualizaciones de las agentes educativas debe provocar un proceso de capacitación que se oriente a la resocialización. De igual manera, la inexistencia de un fondo o programa que incentive la realización de las adaptaciones en la infraestructura excluye a la niñez que requiere matricularse.

Palabras clave: educación de la primera infancia, educación inclusiva, necesidades educacionales

Abstract: The article is carried out by the need to visualize the inclusive processes from the perspective of assistants, educators and directors belonging to the Integra Foundation, the National Board of Kindergartens (JUNJI) and gardens managed by the Municipality Vía Transferencia de Fondos (VTF) of the Copiapó commune of the Third Region of Chile, institutions that, by guidelines, are declared inclusive and recognized for working with the most vulnerable child population in Chile. The main objective of this research was to analyze the inclusive processes of preschool education in the Copiapó commune. He used the qualitative approach with an interpretive paradigm, through semi-structured interviews applied to nine educational agents in 2019, addressing meaning categories, learning methodologies, and impediments and facilitators. The results indicate that, although the educational agents use learning methodologies that favor the participation of each child, there is a conceptual lack of knowledge of inclusive education and disability. Regarding obstacles, there is little evidence of training on disability for educational agents, insufficient didactic material, outdated professional training and little infrastructural accessibility in the gardens. Regarding facilitators, external networks, enrollment management and the relationship of the educational agents with the family stand out. It is concluded that the incoherence of conceptualizations of the educational agents should provoke a training process that is oriented towards re-socialization. Similarly, the absence of a fund or program that encourages the implementation of adaptations in the infrastructure excludes children who require enrollment.

Keywords: early childhood education, inclusive education, disability, educational need.

\footnotetext{
1 Egresada de Trabajo Social de la Universidad de Atacama, Copiapó, Chile. Candidata en Máster de Investigación en Metodología Cualitativa en Salud de la Universidad de Atacama, Copiapó, Chile. Dirección electrónica: fernandarubilarbugueno@gmail.com. ORCID: https://orcid.org/0000-0002-8367-0717

${ }^{2}$ Académica del Departamento de Trabajo Social de la Universidad de Atacama, Copiapó, Chile. Asistente Social, Magíster en Trabajo Social y Políticas Sociales. Dirección electrónica: daniela.guzman@uda.cl. ORCID ID: http://orcid.org/0000-0001-7905-309X
}

Artículo recibido: 25 de junio, 2020

Enviado a corrección: 15 de setiembre, 2020

Aprobado: 19 de octubre, 2020 


\section{Introducción}

Cuando se busca visibilizar, desde las experiencias individuales, los procesos sociales, es importante incluir en el análisis todas las aristas que confluyen en la construcción de una sociedad inclusiva. Considerando que la educación es un derecho, su acceso, operatividad y calidad debe estar resguardada y constantemente monitoreada, en especial, para asegurar su adecuado cumplimiento en aquellos grupos minoritarios que se encuentran en estado de desprotección y/o vulnerabilidad. Tal como lo menciona Muñoz (2018), "la Educación Inclusiva debe contemplar el derecho de toda persona a la educación, sin ningún tipo de exclusión, a través de constantes reflexiones y análisis sobre las prácticas pedagógicas tendientes a mejorar los aprendizajes en ambientes estimulantes" (p.80). Es cada vez más necesario conocer y analizar constantemente las prácticas pedagógicas que se implementan en distintos contextos educativos, y que se proyectan como facilitadoras de la inclusión.

En términos generales, se entiende por educación inclusiva aquella que acoge a toda la diversidad humana, reconociendo que todas las personas tienen potencialidades y habilidades distintas. Específicamente, su propósito es incluir a todas las personas que, a causa de alguna característica distintiva, quedan excluidas de la educación (Corrales, Soto y Villafañe, 2016). Dentro de aquella diversidad se encuentran las personas con discapacidad.

El Estado Chileno ha impulsado políticas y reformas sobre la inclusión de las personas con discapacidad, una de ellas es la Ley 20.422 (2010), que establece Normas Sobre Igualdad de Oportunidades e Inclusión Social de Personas con Discapacidad, orientada a lograr una plena inclusión social asegurando el disfrute pleno de sus derechos. En relación con la inclusión de niños y niñas, ya en 1990, al retorno de la Democracia, la Convención de los Derechos del Niño, a la que se adscribe el país, establece, en su Artículo 28, el derecho del niño a la educación en igualdad de condiciones y oportunidades (Decreto $N^{\circ} 830,1990$ ), respaldando, con ello, que la educación para las personas con discapacidad tiene un papel fundamental en materia de derechos humanos. Esto se retoma en la Convención de personas con Discapacidad, ratificada en el decreto 201 (2008), en donde se reconoce este compromiso, especificando que los niños y las niñas con discapacidad deben gozar plenamente de todos los derechos humanos y las libertades fundamentales en igualdad de condiciones, y recordando los compromisos que a este respecto asumieron los Estados Partes en la Convención sobre los Derechos del Niño. En especial la Organización Mundial de la Salud (2013) declara que, tanto niñas como "niños con discapacidad tienen menos probabilidades de iniciar la escuela y menores índices de permanencia escolar. Se estima que un tercio de 
todos los niños en edad escolar primaria que no asisten a la escuela, son niños con discapacidad" (p. 17).

En el estudio de calidad en educación parvularia menciona que las matrículas de niños y niñas con discapacidad en la educación parvularia ha aumentado consecutivamente. No obstante, indica que la permanencia de la asistencia es menor en comparación de quienes no tienen discapacidad, resalta que las agentes educativas no reportan los motivos (Centro de Políticas Comparadas de Educación, 2015). Para esta investigación, las agentes educativas comprenden técnicos, educadoras y directoras.

Así también, un estudio de la Junta Nacional de Jardines Infantiles (2017) establece que una de las principales razones del retiro de párvulos en los jardines infantiles es la dificultad del proceso de adaptación que tienen algunos niños y niñas con alguna discapacidad, y sobre todo, se destaca que este acontecimiento no es evaluado ni abordado por las agentes educativas, lo cual, finalmente, propicia su expulsión.

En Chile existen dos grandes instituciones que son las responsables de la administración de los jardines infantiles del país. Una de ellas es la Fundación Integra, institución de derecho privado sin fines de lucro que pertenece a la Red de Fundaciones de la Presidencia y la Junta Nacional de Jardines Infantiles (JUNJI), institución de carácter público, perteneciente al gobierno de Chile y que se caracteriza por supervisar establecimientos de administración directa, empadronamiento de salas cuna y jardines infantiles particulares y fiscalización a establecimientos de la sociedad civil a los que se transfieren fondos estatales. Esta última imparte y financia una modalidad llamada Vía Transferencia de Fondo (VTF), que es ejecutada por Municipios. Ambas instituciones son reconocidas por atender a familias que se encuentran en contextos vulnerables y que se declaran inclusivos, lo cual implica que cada niño y niña de determinada comunidad aprendan independientemente de sus condiciones personales, sociales o culturales, incluidos quienes presentan discapacidad (Elige Educar, 2019). Cabe precisar, que este estudio abordó estos contextos educativos mencionados.

La educación Parvularia en Chile es considerada fundamental para promover la igualdad de oportunidades. La educación parvularia impartida por centros educativos tiene efectos positivos y significativos sobre el desarrollo de habilidades socioemocionales y cognitivas de niños y niñas, pues es una forma de proveer a la estimulación temprana base para los futuros aprendizajes (Fondo de Investigación y Desarrollo en Educación, 2018). De ahí nace la importancia de profundizar en procesos inclusivos de niños y niñas con discapacidad. 
El estudio tuvo por objetivo general: Analizar procesos inclusivos de niños y niñas con discapacidad del nivel Medio Mayor de jardines infantiles de Fundación Integra, JUNJI y VTF de la ciudad de Copiapó. De manera particular se plantearon tres objetivos específicos: analizar significados de educación inclusiva, aprendizaje y discapacidad que tienen las agentes educativas; determinar las metodologías de enseñanzas utilizadas por las educadoras que favorezcan la inclusión de niños y niñas con discapacidad; y finalmente, identificar, desde las agentes educativas, facilitadores y obstaculizadores en el desarrollo de procesos inclusivos de niños y niñas con discapacidad.

\section{Referente teórico}

\subsection{Construcción y reconstrucción de la realidad: significados, intersubjetividad y vida cotidiana}

Las personas, como parte de un mundo social, están permeadas por su biografía de vida, lo que las hace únicas y particulares en el mundo. Su crianza, educación, intereses, creencias, entre otros son aspectos propios que conforman la identidad y personalidad de cada una de ellas. De modo que, todo lo anterior, influirá en el significado que le otorga a la realidad, y posteriormente a sus acciones, entendiendo por acciones aquellas que adoptan un carácter relacional consciente, es decir, tiene un significado y un sentido para la persona (Leal, 2009).

De este modo, y focalizándose en este ámbito de interés, podemos decir que las acciones de las agentes educativas dependen de las significaciones que tengan sobre discapacidad, aprendizaje, educación inclusiva, metodologías de aprendizajes inclusivos, y cómo también influirá en la comprensión de procesos inclusivos. Sin embargo, en la cotidianidad las personas suelen efectuar actos repetitivos de forma inconsciente en el mundo social, impidiendo que la misma pueda pensar en su accionar (Mora, 2009). Si bien, estos actos se diferencian de las rutinas de aprendizaje, en ocasiones, estas se tornan repetitivas, desarrollándose siempre de igual manera, con información poco actualizada, vacíos de dinámicas u otros. De manera que, produce que el alumnado muestre poca autonomía, proactividad e indiferencia, volviéndose sólo receptivo de las enseñanzas dictadas por quién realiza docencia (López, 2010).

Generalmente, en las aulas de clases se desarrollan interrelaciones, comunicaciones, interpretaciones que específicamente se dan entre las agentes educativas y párvulos, esto se entiende como un acuerdo intersubjetivo, puesto que ambos trabajan en la construcción del proceso de enseñanza-aprendizaje (Mora, 2009). Estas interacciones pedagógicas son 
definidas como el "proceso de interacción pedagógica, social, dialógico, lúdico, consciente, intencional, sistemático, destinado a generar experiencias de 'buen aprendizaje', que al tiempo que transmita conocimientos, posibilite el desarrollo de las potencialidades humanas en el ser, hacer, conocer y convivir" (Escobar, 2011, p. 60).

\subsection{La educación un derecho de la primera infancia}

Con la ratificación de la Declaración Universal de Derechos Humanos en 1948, a la que se adscribe Chile, se produce un cambio paradigmático, comprometiendo a Gobiernos adscritos a actuar de forma determinada para promover, proteger y garantizar derechos económicos, sociales, culturales, civiles, políticos de todas las personas (Resolución 217, 1948). Posteriormente, se ratifica la Convención de los Derechos del Niño en el año 1990, que tiene como compromiso garantizar el respeto y la protección de los derechos de cada infante (Decreto 830, 1990).

Consecutivamente, se ratificó la Convención de las Personas con Discapacidad en el año 2008. Esta legalización se constituye como un avance significativo para el país, puesto que se incorpora la obligación de generar cambios orientados a la reconstrucción social. Lo anterior se manifiesta en su Artículo 9, donde declara asegurar el acceso del entorno físico, transporte, información, comunicaciones, tecnologías y otros servicios e instalaciones abiertos al público o de uso público, tanto en zonas urbanas como rurales (Decreto 201, 2008). También se da cumplimiento en la aplicación de la Ley 20.422 (2010), que promueve, protege y garantiza a las personas con discapacidad un disfrute pleno del acceso a la sociedad. Del mismo modo, compromete al Estado a tomar decisiones pertinentes para asegurar la realización de ajustes necesarios, las cuales se constituyen por ser adecuaciones y modificaciones, que figuran como soluciones técnicas en espacios infraestructurales cuando es requerido por un caso particular, lo cual permite garantizar a las personas con discapacidad el goce y el ejercicio pleno en igualdad de condiciones.

En relación con la Convención mencionada con anterioridad, alude que el término adecuado para referirse a este grupo es de personas con discapacidad o personas en situación de discapacidad (PsD) (Decreto 201, 2008), ya que "no existen las capacidades diferentes o necesidades especiales. Todos y todas tenemos las mismas capacidades (potenciales, reales, en desarrollo) y necesidades (amar y ser amados, comer, vestirse, tener salud, sobrevivir) (Sección de Participación, Género e Inclusión, 2017, p.2). 


\subsection{Educación parvularia una estrategia para la igualdad}

Actualmente, en Chile, en el marco de la reforma educacional e inclusiva, el Decreto Exento 83 (2015) promueve la diversificación de la enseñanza en educación parvularia y básica, y aprueba criterios y orientaciones de adecuación curricular. Este decreto busca que las escuelas regulares y particulares desarrollen todas las medidas necesarias para dar respuestas a los diversos requerimientos y necesidades educativas que presente el estudiantado a fin de garantizar su acceso, participación y progreso en los aprendizajes del currículum (Ministerio de Educación de Chile, 2015). Las adecuaciones curriculares en la educación parvularia son aquellas estrategias de intervención en la planificación que pretenden acercar el aprendizaje de cada niño y niña, sin importar las diferencias físicas, cognitivas y socioculturales. Estas adaptaciones también permiten su concreción y logró con las normas que dicta la Ley 20.422 y los tratados internacionales vigentes del país, como la Declaración Universal de los Derechos Humanos, Convención sobre los Derechos del Niño y la Convención de las Personas con Discapacidad mencionados con anterioridad (Subsecretaría de Educación Parvularia de Chile, 2018).

Las bases curriculares de la educación parvularia indican que los establecimientos que imparten educación en la primera infancia deben responder a requerimientos de formación como, por ejemplo, ser inclusivos, interculturales, que acepten y promuevan la diversidad, que las decisiones, planificaciones y ejecuciones relacionadas con la educación de cada niño y niña considere los enfoques de género y de derecho. Esto, accede al mejoramiento de la calidad educativa y a la posibilidad de responder a las necesidades de aprendizaje y desarrollo de todos y todas (Ministerio de Educación de Chile, 2018). Estos requerimientos se observan en el desarrollo y preparación de las clases.

La planificación, como definición, es el diseño y ejecución de las instancias educativas que permite ordenar, estructurar y guiar el trabajo educativo en tiempos específicos. Las planificaciones deben tener en cuenta aprendizajes previos, edad y diversidad de los párvulos, tanto niños como niñas y a su vez complementarlas con "estrategias en que se desarrollen iniciativas que les motiven, lo que fortalecerá habilidades asociadas a las funciones ejecutivas, como asociar ideas, orientar la acción hacia una meta, planificar, definir objetivos, poner en juego su memoria" (Ministerio de Educación de Chile, 2018, p.48). Respecto al tiempo de duración de la planificación, esta puede ser de largo a mediano plazo (anual, trimestral, semestral), debe tener una secuenciación y ordenamiento general de cada objetivo (Superintendencia de Educación de Chile, 2017), los que corresponden a una intencionalidad 
pedagógica que se busca cumplir. Cabe destacar que los objetivos de aprendizaje "buscan alcanzar en la formación personal y social, que los niños y las niñas a través del desarrollo de la autonomía, la conciencia gradual de sus posibilidades corporales y motrices (...) puedan enfrentar diversos desafíos de aprendizaje" (Ministerio de Educación de Chile, 2018, p.39). Los objetivos logran mayor impacto con estrategias inclusivas orientadas a:

(...) ofrecer a los niños y niñas oportunidades efectivas de aprendizaje, disminuir las barreras para su participación garantizando una educación de calidad en condiciones que se ajusten a su realidad. Conlleva a identificar y responder a la diversidad de necesidades de todos los niños y niñas. (Niño, 2016, p. 29)

Así también, el juego es considerado, al momento de planificar, como una herramienta pedagógica para el aprendizaje en la primera infancia, donde tiene un fin y un impacto, ya que responde a las motivaciones internas de niños y niñas (Ministerio de Educación, 2018). Lo anterior, es complementado con rincones o sectores de aprendizaje, que se caracteriza por ser una estrategia pedagógica que hace posible la participación de niños y niñas en la construcción de sus propios conocimientos de manera cooperativa e interactiva (Calvillo, 2013). Un factor que impacta el tránsito a una educación inclusiva es "el poder de autorregulación e inclusión que tienen los rincones y el trabajo en grupo". (Sánchez, 2019, p.4)

Espejo y Sarmiento (2017), en su propuesta de diseño e implementación de estrategias de enseñanza-aprendizajes activas, incorporó la retroalimentación como una estrategia de evaluación que potencia la calidad del aprendizaje. Por ejemplo, en el trabajo realizado a través de la metodología de los rincones, la evaluación se focaliza en valorar el aprendizaje de cada niño o niña, no prestando atención únicamente al resultado final de cada periodo lectivo, sino evaluar todo el proceso. Para ello, la observación continua y exhaustiva es la mejor herramienta para los procesos de retroalimentación que completan las evaluaciones de los aprendizajes finales de cada niño y niña (Sánchez, 2019).

Sin embargo, aún es posible presenciar en la educación obstaculizadores que impiden el desarrollo pleno del alumnado. Existe experiencia acumulada respecto a las distintas barreras a las que se enfrentan las personas en situación de discapacidad, y el espacio educativo no es la excepción. Corrales, Soto y Villafañe (2016) mencionan que las barreras en las culturas escolares limitan el aprendizaje y la participación del estudiante. Por tanto, las dificultades de una persona en el aprendizaje o la discapacidad no pueden entenderse, sin antes considerar el contexto donde se desenvuelven. En este sentido, los autores identifican 
cuatro tipos de obstaculizadores: barreras de acceso, barreras de participación, barreras de gestión y barreras culturales.

Castillo y Miranda (2018) en su estudio efectuados a futuros docentes, indican que sus prácticas cotidianas no son el fiel reflejo de su imaginario, puesto que los establecimientos mantienen las enseñanzas y aprendizajes sin espacio para la innovación, ya sea, por la falta de tiempo, escasez de recursos, escaso apoyo de la administración educativa u otro. Además, reconocen una debilidad en la formación pedagógica, que no los preparan para enfrentar los desafíos de la inclusión y atención a la diversidad.

En cuanto a los facilitadores en educación, Grande y González (2015) aluden que las condiciones, procesos y prácticas educacionales se constituyen como puntos fuertes para el proceso de inclusión, que ponen al servicio el aprendizaje y la participación. Algunas estructuras, recursos o estrategias que facilitan una educación inclusiva son: el ambiente de calidad, formación docente, actitudes y responsabilidad docente, comunidades de aprendizaje, apertura a la comunidad, agrupamientos diversos de niños y niñas, y el trabajo colaborativo. En suma, las estrategias educativas centradas en el aprendizaje y desarrolladas a partir de flexibilidad metodológica y actividades diversas permiten el conocimiento de diferentes maneras. Complementario a lo anterior, en el estudio de Sirkko, kyronlampi y Puroila (2019) efectuado en Finlandia, declara que algunas herramientas pedagógicas habilitan los aprendizajes de niños y niñas. Resaltan, que los juegos y materiales con impacto pedagógico apoyan la participación en aula, proporciona control y disciplina en los infantes, así como también, fortalece el sentido de pertenencia en aula. Por último, se evidencia que los tiempos de descansos y los juegos libres posibilitan interacciones sociales entre pares.

\section{Metodología}

\subsection{Enfoque}

El estudio adoptó un enfoque cualitativo mediante el paradigma interpretativo. Este paradigma entiende que las personas no descubren el conocimiento, más bien lo construyen. Se basa en la comprensión profunda de la realidad y de cómo se genera el conocimiento. Básicamente, esto se logra desde la interacción entre el sujeto de estudio y quien investiga. Asimismo, se caracteriza por enfatizar siempre lo subjetivo, conocer para comprender lo que se revele en el estudio investigativo (Ricoy, 2006). De igual manera, fue posible obtener "detalles complejos de algunos fenómenos, tales como sentimientos, procesos de 
pensamiento y emociones" (Strauss y Corbin, 2002, p.21), lo que posibilitó una interpretación pura, profunda y particular.

\subsection{Unidades de análisis}

La investigación se desarrolló entre abril y diciembre del año 2019. La población de este estudio comprendió a directoras, educadoras y técnicas en educación parvularia de las instituciones Fundación Integra y Junta Nacional de Jardines Infantiles (JUNJI), junto a este se encuentra la modalidad Vía Transferencias de Fondos (VTF) ejecutadas, específicamente, por municipalidades. Las participantes fueron nueve informantes claves del sistema educativo, agrupadas de la siguiente manera: directoras, educadoras y técnicas de educación parvularia, todas perteneciente a la comuna de Copiapó.

Cabe destacar que los jardines seleccionados fueron confidenciales para ser susceptibles de protección. Así también, se establecen criterios de inclusión para esta investigación, los cuales fueron: mujeres, con al menos cuatro años de servicios en las instituciones Fundación Integra, JUNJI y VTF, que realicen sus funciones educacionales en el nivel Medio Mayor y tengan a cargo, al menos, tres niños o niñas con discapacidad.

El procedimiento de selección se constituyó en dos fases, la primera fue contactar a las Direcciones Regionales de Atacama de Fundación Integra y de JUNJI, y al Departamento de Administración de Educación Municipal (DAEM), que se encarga de la ejecución de VTF, todas ubicadas en la ciudad de Copiapó, Chile. Luego del consentimiento de cada una de las direcciones, se les pidió un catastro con respecto a la cantidad de niños y niñas con discapacidad por nivel y jardín. Luego de la selección del nivel y de los establecimientos a estudiar, se dirigió a cada uno para conocer a las participantes del estudio de los niveles Medio Mayor y establecer acuerdos sobre horarios para el desarrollo de las entrevistas. La segunda fase fue en terreno realizado en cada contexto educacional, a cada una de las informantes se le explicó el consentimiento informado, y una vez firmado se podía dar paso al desarrollo de las interrogantes que se relacionaban directamente con las categorías de análisis.

\subsection{Técnicas de recolección}

La técnica utilizada para la recolección de datos fue: entrevista semiestructurada. Las preguntas se construyeron en base a temas relevados en la literatura. Se efectuaron dos entrevistas pilotos para perfilar y perfeccionar el proceso de entrevista; la primera fue a una 
técnica en educación parvularia y la segunda, a una educadora de párvulos, ambas de la Universidad de Atacama.

Las preguntas desarrolladas se relacionaron con el significado de educación inclusiva, discapacidad y aprendizaje, en consecuencia, metodologías de aprendizajes que favorecen la inclusión de niños y niñas, y finalmente se abordó facilitadores y obstaculizadores de procesos inclusivos. Cabe precisar que las entrevistas fueron registradas a través de una grabadora de voz, lo cual fue especificado en el consentimiento informado.

\section{Procesamiento de análisis}

El análisis de la información inicia con la transcripción de las entrevistas realizadas, las que fueron organizadas en unidades hermenéuticas. Se ejecutó de manera descriptiva la identificación de categorías madres y subcategorías, asociando los relatos a las dimensiones preestablecidas. Coincidente con lo planteado por Morris (1994), el proceso de análisis se instruye con disponer el material, apartando el contenido en virtud de dicho material, de modo que se pueda agrupar todo que parezca guardar relación, la bastante como para ser considerada similar, mientras que otros conjuntos de datos acceden a otros grupos. Es en este momento donde se pone en práctica la creatividad, la capacidad de integración y síntesis (Cáceres, 2003). Esto deriva en la delimitación de categorías y subcategorías (Figura 1): 
Figura 1

Categorías y subcategorías de análisis cualitativo, del año 2019.

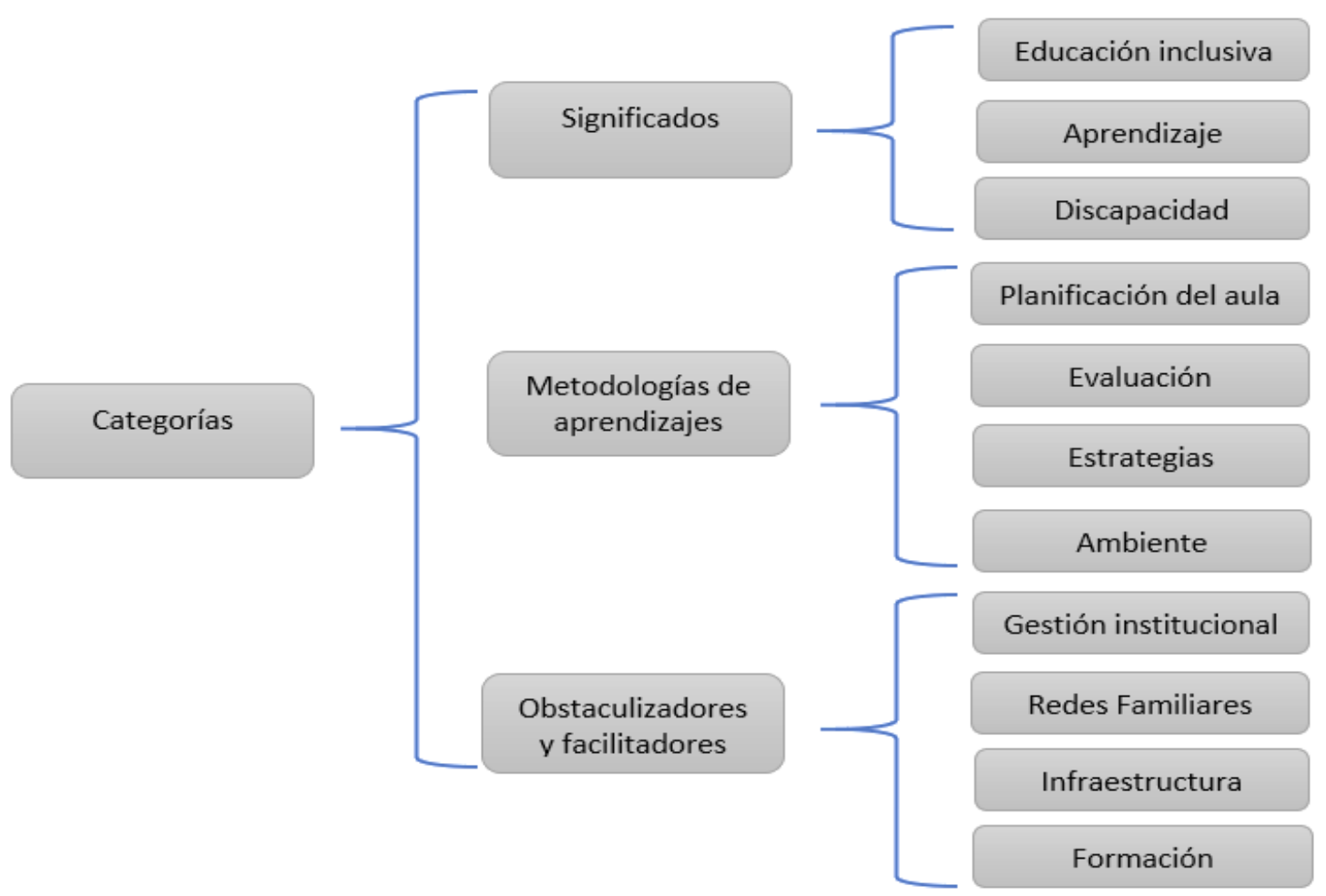

Fuente: Elaboración propia, del año 2019

\section{Resultados}

Para responder al objetivo general, primeramente, se analizaron los significados que tienen las agentes educativas sobre educación inclusiva, aprendizaje y discapacidad, seguidamente se determinó las metodologías de aprendizaje utilizadas por las educadoras de párvulo en sus procesos de enseñanza-aprendizaje. Finalmente, se identificaron facilitadores y obstaculizadores en el desarrollo de los procesos inclusivos de niños y niñas con discapacidad de los jardines infantiles de JUNJI, VTF y Fundación Integra de la comuna de Copiapó de la región de Atacama, Chile.

\subsection{Categoría: Significados}

\subsubsection{Subcategoría: Educación inclusiva}

Los significados construidos socialmente y expresados en la vida cotidiana van a depender de la biografía de vida y las experiencias personales de las agentes educativas, que 
influyen en sus formas de pensar y actuar. En este sentido, es crucial mencionar que las agentes educativas visualizan la educación inclusiva como una educación cuya responsabilidad es aceptar e integrar a niños y niñas con discapacidad, es decir, entienden que la meta del jardín infantil es incorporar a alguien que está siendo excluido.

(...) porque inclusión es como heee, incluir a aquellos niños que por distintas razones o circunstancias la sociedad o uno mismo deja de lado, integrarlos... heee a ver, es como lo mismo, no sé si me explico, integrarlo es integrarlo y como que inclusión es lo mismo, es tratar de que todos seamos parejos e iguales. (ET4)

(...) integrar a los niños que tienen discapacidades diferentes, tal cual, como se trabaja en aula con los niños "normales", heee integrarlos, heee de todas maneras en tanto social, en trabajo que uno realiza uno en aula (...). (ET3)

De acuerdo con las citas, las agentes educativas asimilan o confunden el concepto de inclusión con integración, prevaleciendo el concepto de integración en sus discursos. Se infiere que los discursos son muy contradictorios con la filosofía de una educación inclusiva, ya que se entiende que el medio es el que debe encontrarse accesible para que todas las personas, familias y comunidades puedan participar sin restricciones (Corrales, Soto y Villafañe, 2016).

Con respecto al significado que le otorgan las agentes educativas a la educación inclusiva, es posible relacionarlo con lo planteado por Alfred Schutz, este autor menciona que las acciones de origen subjetivo se presentan de manera cotidiana a través de conductas objetivas, y estas últimas son interpretadas volviendo nuevamente a adoptar un carácter subjetivo (Leal, 2009). En otras palabras, se puede interpretar que las acciones de las agentes educativas están relacionadas directamente con una metacognición, la cual se presenta en la vida cotidiana mediante conductas objetivas, sin embargo, estas vuelven a ser reinterpretadas por otras personas de manera subjetiva.

\subsubsection{Subcategoría: Discapacidad}

Se pudo constatar una tendencia en la utilización de conceptos como "normales", "capacidades diferentes" y "minusválido" en los discursos, que demuestran la coexistencia de conceptos contradictorios al lenguaje inclusivo. Asimismo, este suceso direcciona a comprender que las entrevistadas visualizan a los niños y niñas con discapacidad distintos o distintas a la norma, ignorando que cuando una persona con discapacidad se encuentra en 
interacción con el medio es cuando las limitaciones aparecen e impiden su participación plena. Las recomendaciones que realiza la Sección de Participación, Género e Inclusión (2017) en el uso del lenguaje inclusivo plantea que no existen capacidades diferentes ni necesidades especiales, puesto que todos tenemos las mismas capacidades y necesidades, solo que el entorno imposibilita que las vivencias de todos y todas no puedan generarse en igualdad de condiciones.

(...) Nicolás usa pañales y estuve yo veinte minutos o treinta minutos sentada con el recién conociéndolo, para tratar de cambiarle pañales porque estaba defecado y no había caso, no había caso no, no, no, lloraba, lloraba, lloraba, entonces llamé a la mamá y le pregunté ¿Qué es lo que sucede? ¿Cómo usted lo hace? o ¿Qué es lo que le pasa a él? Entonces ella viene y me dice tía a Nicolás le gusta cambiarse pañales en un lugar solo, cuál fue la estrategia que utilizamos con él y hasta el día de hoy la utilizamos súper bien, es él va al baño de minusválido que está al lado y ahí le cambiamos pañales (...). (EE1)

(...) No hacemos nada separado y ellos se integran a ese proceso, son como niños "normales" (...) (EE2).

Se puede señalar, también, que prevalece una tendencia en relación con la sobreprotección de los niños y niñas con discapacidad, este hecho es justificado en el temor de que experimenten algún daño físico o emocional.

(...) con el Agustín tengo una sobreprotección, así como, heee que le vayan a pegar así o voy con el niño y le digo "no, pero es que el Agustín lo necesita, si no se va a poner a llorar" término no sé de convencer al otro compañero, ya, para que le de sus juguetes, para que no llore o empieza a llamar a su mamá (...). (ET4)

La cita anterior demuestra muy bien la sobreprotección hacia Agustín, ya que es posible presenciar que es capaz de convencer a otros niños o niñas sin discapacidad de no utilizar ciertos juegos que claramente son los favoritos del niño, por ende, el resto de los infantes están siendo excluidos. 
En congruencia, se infiere que las experiencias personales de las agentes educativas influyen en los significados, decisiones y acciones que tendrán con una persona con discapacidad (Leal, 2009).

\subsubsection{Subcategoría: Aprendizaje}

El manejo y conocimientos óptimos de enseñanzas y aprendizajes de las agentes educativas es debido a la incorporación de estas en toda formación profesional o técnica. Las entrevistadas declaran que los aprendizajes se desarrollan mediante el vínculo y es entendido cuando cada niño y niña reflejan lo aprendido. Inclusive, manifiestan que cada trabajo efectuado en aula es igual para todos y todas, pero la mediación es diferente, porque va a depender de las características propias de cada párvulo.

(...) por ejemplo, yo nunca he hecho una adecuación en mi planificación, es todo, para todos igual, ya, lo que se trabaja diferente es la mediación, como uno, como agente o como educadora, viene a ese aprendizaje para que ese niño con necesidades educativas especiales pueda aprenderlo de igual forma que el resto, de esa forma trabajamos (...). (EE1)

Según Muñoz (2018), el aprendizaje se asocia al respeto por los diferentes ritmos de aprender y a la incorporación de metodologías y estrategias inclusivas. Coincidentemente, desde los discursos de las entrevistadas, se destaca el respeto por los diferentes ritmos de aprendizaje. Para ello, se utilizan diferentes ambientes o rincones para complementar sus prácticas inclusivas.

(...) son todos los espacios iguales (...) en el espacio del arte ponte hay diez niños y los diez quieren trabajar y entre ellos está el niño con, con, con pincel, se propicia que haya diez pinceles (...). (EE2)

El concepto de diversidad se encuentra incorporada en todos los procesos de enseñanzasaprendizajes de los niños y niñas. Las entrevistadas entienden el concepto de diversidad como la aceptación y valorización de las características propias de cada persona.

(...) Diversidad para mi es la inclusión, ya o sea para mi yo como dije también se los traspaso a todos los apoderados, a mis tías a todos. La inclusión, para mí es un proceso que netamente tiene que ver con la diversidad, si tú no eres capaz de entregar aprendizajes en este caso que sean diversificados, no lo obtienes (...) (EE1). 


\subsection{Categoría: Metodología de aprendizaje}

\subsubsection{Subcategoría: Planificación del aula}

Vigorosamente, las agentes educativas rescatan los aprendizajes previos, la edad y el interés del párvulo como fuente de conocimiento para la planificación del aula y parte de las adecuaciones metodológicas. Además, manifiestan que lo teórico es de gran importancia a la hora de efectuar planificaciones, ya que, después de todo, guía directamente el quehacer profesional de las entrevistadas. Se destaca la utilización de instrumentos pedagógicos que contienen referentes teóricos, como las bases curriculares, referente curricular propio de la institución y el Diseño Universal de Aprendizaje (DUA).

(...) trabajamos con el DUA en nuestras planificaciones que es el diseño universal de aprendizaje, entonces con eso nosotras le ofrecemos a los niños que tiene necesidades educativas especiales distintos focos donde ellos puedan aprender de acuerdo a sus intereses y de acuerdo a lo que ellos quieren hacer (...). (EE1)

Principalmente las bases curriculares de la educación Parvularia, el referente curricular de la institución (...). (EE2)

Coincidentemente, las bases curriculares de la educación parvularia mencionan que el utilizar los aprendizajes previos, edad y considerar diversidad, y potenciarlo con estrategias motivadoras permiten favorecer el desarrollo del aprendizaje y habilidades de los niños y las niñas (Ministerio de Educación de Chile, 2018). La flexibilidad también es un factor relevante a la hora de planificar, puesto que constantemente las agentes educativas realizan modificaciones en los objetivos de aprendizajes, contenidos de aprendizajes y la temporalidad de las planificaciones, esto es debido a que los niños y niñas van presentado o cambiando sus necesidades.

(...) si yo hago una planificación, pero a los 15 días los niños no están ni ahí con mi planificación o hay algo que no les gusta y ellos te dicen jhay tía no! Yo creo que ellos se van a otros espacios, que buscan otras cosas ¡la témpera!, entonces yo tengo que modificar, conversar con mi equipo de sala y decir "sabes que, estamos viendo esto" ¿Qué podemos hacer? (...). (EE2) 
La flexibilidad de la respuesta educativa debe procurar que el ajuste realizado sea equivalente en calidad, favorezca el acceso, permanencia y progreso de la totalidad de estudiantes, especialmente resguardar aquellos que se encuentran en situación de mayor vulnerabilidad (Ministerio de Educación de Chile, 2015).

\subsubsection{Subcategoría: Evaluación}

En coherencia con lo planteado por las bases curriculares del Ministerio de Educación de Chile (2018), la totalidad de las entrevistadas mencionan que sus evaluaciones de aprendizaje son cualitativas y descriptivas, puesto que les permite redactar los aprendizajes y observaciones de cada niño y niña, esto es complementado con bitácoras, fotos y registros de observaciones que permiten un análisis integral del aprendizaje. Se destaca que las familias también son parte del proceso evaluativo, encontrándose activas en los aprendizajes de niños y niñas. Este método permite registrar avances y aspectos por mejorar en las familias, información reportada y trabajadas en las reuniones pedagógicas.

(...) nosotros realizamos una evaluación cualitativa, describir acciones que hacen el niño (...) También evaluamos los contextos de aprendizaje ¿Cómo estuvo el espacio? ¿Cómo estuvieron los materiales? ¿Qué nos faltó en la planificación? Esa se evalúa un contexto semanal y la familia también evalúa los desafíos en sí, se le pregunta ¿le preguntó a su hijo que hicimos hoy día? O ¿Qué hicimos ayer? (...). (EE2)

La evaluación es una herramienta que recoge y analiza la información de procesos de aprendizajes, la cual sirve para tomar decisiones que contribuyan a mejorar procesos y resultados de aprendizajes (Subsecretaría de Educación Parvularia de Chile, 2018). Las entrevistadas revelan la importancia de la evaluación como un insumo para fortalecer el proceso y mejorar las planificaciones. Asimismo, las agentes educativas consideran la evaluación como una herramienta de retroalimentación, que permite valorar avances en relación a las decisiones metodológicas adoptadas por el equipo.

(...) intereses de los niños, primero que nada, evaluaciones anteriores, ya hem...tengo que seleccionar en los objetivos de aprendizajes de acuerdo a los intereses y características de los niños específico del nivel y ver el tema de los referentes curriculares nuestro PEI y obviamente nuestras bases curriculares, todo eso se toma en cuenta. (EE1) 


\subsubsection{Subcategoría: Estrategias}

Cabe destacar que las entrevistadas responden a este apartado con numerosos ejemplos, de modo que fueron analizados y se identificaron tres estrategias o tácticas que permiten contribuir con el aprendizaje inclusivo en el aula. La primera estrategia es la mediación, esto significa que las agentes educativas deben estar atentas a todas las señales y acciones de niños o niñas, y junto a esto, generar preguntas de aprendizajes que permita responder adecuadamente a sus interrogantes.

(...) oye y ¿de qué color es eso? y me puede responder rojo... ah ya, y... el otro día hicimos una actividad sobre las instituciones de la comunidad, y el observaba "bomberos me decía", si ¿los bomberos que hacen? y ahí nosotros empezamos a hacer preguntas para que él pueda igual tratar de comunicarnos más con nosotros (...). (EE3)

La mediación es una herramienta pedagógica que se utiliza en el proceso de aprendizaje, debe ser lúdica y dialógica, consciente e intencional, debe generar experiencias en el aprendizaje, conocimientos y potenciar las habilidades humanas. El rol de la mediadora es clave, puesto que organiza, describe y agrupa el mundo junto a cada niño y niña (Escobar, 2011). Las entrevistadas indican que la mediación es el conducto entre los niños y niñas y el medio, donde las agentes educativas son quienes deben orientar el aprendizaje para desarrollar las ideas y estimular procesos metacognitivos.

La segunda estrategia identificada es el juego. Pedagógicamente es comprendido como una forma de incentivar el aprendizaje colectivo y colaborativo, el cual tiene un fin y un impacto (Ministerio de Educación de Chile, 2018). El juego es desarrollado mediante cuentos, canciones y dinámicas grupales, y como estrategia permite vencer las barreras actitudinales que se crean al momento de generar aprendizaje inclusivo.

Sí, de hecho, el nivel sabe que ellos...por ejemplo los mismos niños saben que en nuestro nivel hay tres niños con TEA eeeh... quizás no lo conocen como TEA, ¿ya?, pero saben que de cierta manera somos todos diferentes es algo que se trabaja desde un comienzo mediante cuentos, mediante láminas, mediante canciones (...). (EE1)

Las entrevistadas destacan que el juego permite simular ideas y roles, afecto, solidaridad, empatía y respeto entre párvulos.

Finalmente, la tercera estrategia reconocida consiste en efectuar retroalimentaciones inmediatas, estas se tratan de preguntas diarias en relación con cada temática abordada en el 
aula. Lo previo es considerado muy valioso en el quehacer profesional de las agentes educativas, ya que, a través de las retroalimentaciones con base en preguntas de activación, desarrollo y cierre, permite comprender el grado de conocimiento de todos los niños y niñas, y fomentar la participación colectiva.

(...) ponte con un juego ponte tú a "la ronda" ya y después a lo mejor no todos, pero después ¡niños! ¿le gusto el juego? ¿le gustaría jugarlo de nuevo? ¿ ¿Cómo se llamaba? O cuando se relata el cuento, también ¿qué nombre tenía el cuento? Pero a lo mejor si la caperucita no le habría hecho caso al lobo ¿Qué le habría pasado? Ellos van como de acuerdo a su lenguaje cómo es escaso van respondiendo. (EE2)

Se deduce que las retroalimentaciones colectivas, en definitiva, se trata de una serie de procesos intelectuales que efectúan las agentes educativas, orientadas a facilitar una educación inclusiva dentro del aula. Si bien, se orienta a potenciar la capacidad y fortalecer continuamente los aprendizajes, su propósito mayor es fomentar análisis y reflexión en torno al propio proceso de aprendizaje y así autorregularse.

\subsubsection{Subcategoría: Ambiente}

El ambiente es un factor esencial a la hora de impartir las clases y los juegos didácticos. Una óptima ventilación, luz, dimensión del espacio y materiales permitirá a cada párvulo encontrarse a gusto, cómodos y sobre todo, con ganas de seguir aprendiendo (Pedrero, 2012). Así lo consideran las entrevistadas, quienes refieren la importancia de los rincones o sectores de aprendizaje a la hora de organizar el salón de clases, pues permite el desarrollo de diversas actividades de acuerdo con el interés del párvulo en distintos lugares. Los rincones más conocidos son: el rincón de lectura, rincón de la casita, rincón de las matemáticas y ciencias, entre otros. Calvillo (2013) caracteriza los rincones como una estrategia pedagógica que hace posible la participación y la construcción de conocimientos de manera cooperativa e interactiva de quienes participan.

(...) si uno no tiene el ambiente o el espacio educativo con el material suficiente, novedoso, significativo, el niño no le va a gustar nada (...). (EE2)

(...) tenemos también el rincón del lenguaje que le tenemos librero, una alfombra y tenemos cojines y ahí los niños se sientan y todo, le tenemos el de Asperger le tenemos una pizarra con tiza, témpera, hojas para que estén felices y el de matemáticas le tenemos elementos concretos donde ellos pueden ir armando (...). (ET4) 
Sánchez (2019) declara que el trabajo realizado a través de la metodología de los rincones focaliza su evaluación en valorar el aprendizaje del niño o niña visualizando el proceso y no prestando atención únicamente al resultado final de cada periodo lectivo. Para ello, la observación continua y exhaustiva es la mejor herramienta. Mediante ella, se adapta la ayuda que cada niño o niña necesita de tal modo que, individualmente, construya su aprendizaje. Muñoz (2018) indica que para lograr una educación inclusiva las prácticas pedagógicas se deben realizar en ambientes estimulantes, para así potenciar los aprendizajes de cada párvulo.

\subsection{Categoría: Obstaculizadores y facilitadores}

\subsubsection{Subcategoría: Gestión Institucional}

Para las agentes educativas la gestión institucional corresponde a la capacidad de las instituciones de responder a lineamientos inclusivos declarados y, por tanto, a las demandas solicitadas para hacer efectivos, y así asumir, adecuadamente, procesos de aprendizajes.

Es en este sentido que se considera un facilitador la vinculación permanente de los jardines infantiles con redes externas de apoyo, como el Centro Comunitario de Salud Familiar (CESFAM) y el Instituto Teletón, donde ambas vinculaciones sirven para complementar la información de todo niño y niña matriculado en el jardín, sobre todo, para nutrir el trabajo con la población infantil con discapacidad. Asimismo, cuentan con redes internas propias de la institución, que permiten avanzar hacia un trabajo interdisciplinario, constituido por profesionales de la psicología, la educación diferencial y el trabajo social, quienes se encargan de informar y entregar herramientas para poder otorgar una atención oportuna.

En relación con la formación continua, cabe destacar que las instituciones de pertenencia tardan en generar capacitaciones (redes internas), y cuando se efectúan sólo abordan problemáticas comunes en niños o niñas, aspectos transversales y generales que no logra atender la complejidad de las orientadas a fortalecer el proceso de enseñanza-aprendizaje de párvulos con discapacidad. Lo previo es considerado un obstaculizador.

(...) tuvimos una capacitación con...de inclusión, no siempre yo creo falta eso, falta que nos apoye en cuanto para poder atender a los niños (...). (ET1)

(...) lo desconozco, al menos a mí no me han capacitado con respecto a eso. Todo lo que he sabido de Agustín lo he leído en ficha o lo he investigado, pero desconozco si lo han hecho al respecto (...). (EE3) 
De la misma forma, los procesos burocráticos relacionados con la gestión de materiales (juegos) son considerados como obstaculizador por la totalidad de las entrevistadas, debido a que consideran que los materiales didácticos entregados por las instituciones solo responden a un estándar desvinculado de la realidad.

(...) se generan las peleas o las discusiones ¡dos, tres, cuatro, cinco niños quieren lo mismo! entonces se puede uno complicar como poder hacerlo, para que todos estén contentos. (ET4)

(...) pero no pedimos cuantos, porque o si no pediríamos treinta muñecas o treinta coches, porque pelean por dos o tres coches. Pero ellos quizás no se dan cuenta que en la práctica todas van a querer jugar con el coche. (EE2)

Corrales, Hernández y Villafañe (2016) mencionan que las barreras relacionadas con la gestión de un sistema educativo limitan aprendizajes. Por tanto, las dificultades de una persona en el aprendizaje y/o la discapacidad no es posible entenderla, sin antes considerar el contexto donde se desenvuelven.

Por otro parte, se evidencia que cuando los jardines infantiles no cuentan con todas las implementaciones e infraestructuras necesarias para una educación inclusiva, la institución se encarga de derivar a cada infante con discapacidad a un establecimiento que cuente con estas condiciones, lo cual, es considerado como facilitador por las entrevistadas, quienes lo vinculan fuertemente con lo mencionado por la Convención de los Derechos del Niño que garantiza el acceso a la educación (Decreto 830, 1990).

\subsubsection{Subcategoría: Redes familiares}

Ahora bien, la existencia de una relación virtuosa entre las agentes educativas y las familias se considera un facilitador. Estas últimas, no solo cumplen con la función de incorporar a niños y niñas al sistema educativo pre básico, sino que también se evidencian interacciones colaboradoras en reuniones de apoderados en donde hacen entrega de información que aporte en las prácticas inclusivas de las agentes educativas. Asimismo, las familias son informadas sobre los avances y observaciones del párvulo por parte de las agentes educativas.

(...) la familia viene a la comunidad de aula que se hace una vez al mes, también en las tardes, hay comunidades de aprendizajes de aula, las de aula son las del nivel, si, entonces ahí se juntan, los niños salen temprano la familia pueda venir en la tarde, y 
participar ahí en la toma de decisión de que se va a hacer con respecto a alguna temática o que se va a hacer para poder mejorar en algo que está débil en los niños (...). (ED3)

Por tanto, la relación dialógica y constante que existe entre las familias, principalmente representadas por las madres, y las agentes educativas permite no solo obtener información relevante para fortalecer los procesos educativos, sino también promover la confianza y colaboración mutua. Esto se ve reflejado cuando las familias se involucran y participan en las actividades que efectúan sus hijos e hijas dentro de los contextos educativos. También, existe flexibilidad para que las familias puedan permanecer en el jardín infantil si lo requieren, esto sucede principalmente cuando el niño o la niña ingresa por primera vez al jardín infantil y necesita acompañamiento hasta que logre adecuarse.

(...) tenemos que tener una vinculación con la familia, ya, que ellos nos entreguen insumos, que ellos evalúen nuestra planificación, que ellos nos comentan, que ellos nos confeccionen material (...) porque las familias es el primer educador, ya, nosotros somos un complemento a la educación que le entrega la familia (...). (ED2)

\subsubsection{Subcategoría: Infraestructura}

Fue posible hallar que la mayoría de los jardines infantiles investigados tienen más de treinta años funcionando. Pese a ello, se realizan ajustes razonables, tales como nivelación del piso, pasto sintético, cambios de mudadores, iluminación y ventilación en aula, entre otros.

(...) se ha tenido en infraestructura modificar el aula para que tuviera mayor ventilación o mayor iluminación, ampliar la cantidad de tazas, de laboratorios e incorporar una "tineta" para el lavado de los niños (...). (ED1)

Lo anterior da cumplimiento a la Ley 20.422 (2010) donde en su Artículo 8 menciona que se tomarán medidas pertinentes para asegurar la realización de ajustes necesarios o razonables para eliminar las barreras del entorno. El propósito es lograr la autonomía individual e independencia de las personas con discapacidad.

Pese a lo anterior, las entrevistadas manifiestan que los arreglos que se efectúan en los jardines son para equiparar los daños que posee la infraestructura por uso, pero no visualizan cambios que se relacionen directamente con un libre acceso para las personas con discapacidad. Es importante reiterar que el mismo Estado ha ratificado y firmado compromisos con la Convención de los Derechos de las Personas con Discapacidad (Decreto 201, 2008) y 
la creación de Ley 20.442 (2010), la cual ambos tienen la finalidad de garantizar una sociedad justa y equitativa para que todas las personas con discapacidad puedan desenvolverse lo más autónoma y natural posible.

En la misma línea de análisis, aquellos jardines antiguos no pueden matricular a cada niño y niña con discapacidad, porque va depender si el establecimiento cuenta con la accesibilidad e infraestructura para atenderlos.

(...) si llegase un niño con silla de ruedas yo no tengo la infraestructura para recibirlo en el jardín, no tengo rampas para desplazar a un niño con silla de ruedas (...). (ED1)

(...) nos enfocamos solamente en niños que, a ver cómo explicarlo, heee como físicamente están normales sin ningún tipo de discapacidad, entonces se crean los jardines de acuerdo a esos niños, no se crean de acuerdo a niños con necesidades diferentes. (ET3)

\subsubsection{Subcategoría: Formación}

Las entrevistadas consideran un obstaculizador la formación profesional y técnica, ya que mencionan que en su formación académica no abordaron temáticas de inclusión ni de discapacidad. Por tanto, recalcan la importancia de que sean incorporadas en las mallas curriculares, puesto que se requieren de conocimientos óptimos y experiencias fidedignas que contribuyan a sus prácticas inclusivas.

(...) siento que la universidad y a las carreras les falta poner un poco más de inclusión en su enseñanza, porque esto y lo aprendí trabajando, yo salí de la Universidad, pero la universidad fue poquísimo la forma de hablar de estos síndromes o de cosas de la inclusión, súper poco, entonces falta en la universidad estos temas (...). (EE3)

(...) heee la psicología también me pasaban en realidad a mi heee, te pasan no como estrategia "tienes que trabajarlo así", sino como las características de las discapacidades como para poder decir "hay este niño a lo mejor tiene este problema", "porque estará tan callado", "porque no fijara la mirada", "porque come mucho" (...) como para que nosotros también tuviéramos la oportunidad de periciar o saber más menos que problemática puede tener ese niño (...). (ED3) 
El desconocimiento de aquella temática también es posible encontrarlo en los hallazgos de Castillo y Miranda (2018), que refieren que una de las barreras que vivencia el estudiantado con discapacidad es la falta de conocimientos óptimos que prevalece en el cuerpo docente. Igualmente mencionan que el cuerpo docente no está lo suficientemente preparado a nivel de formación para enfrentar los desafíos de la educación inclusiva y la atención de la diversidad. Además, desde los discursos se desprende la tendencia a patologizar las diferencias, en tanto la formación entregada se orienta a caracterizar conductas en niños y niñas que les clasifican como una situación más o menos problemática.

\section{Conclusiones}

Se puede mencionar que el cuerpo directivo, parvularias y técnicas en educación parvularia de los jardines infantiles de JUNJI, VTF y Fundación Integra de la comuna de Copiapó, utilizan métodos de aprendizajes basados en las experiencias y procesos caracterizados por su flexibilidad, contextualización y constante retroalimentación que invita a niños y niñas definir por su propia atracción y elección los medios que utilizarán para la construcción de su aprendizaje. Las agentes educativas implementadoras de estos métodos y estrategias, desde su rol como mediadoras desarrollan desafíos o retos de aprendizajes y fomentan la autonomía en el logro de soluciones. En relación con las metodologías y evaluaciones de aprendizajes, estas se caracterizan por ser cualitativas, flexibles y diversificadas. Asimismo, se identifican tres grandes estrategias para el proceso de enseñanza-aprendizaje: la mediación, el juego didáctico y la retroalimentación, como herramientas pedagógicas que aportan al aprendizaje inclusivo.

Así entonces, desde lo declarativo, se establece coincidencia con los lineamientos de una educación preescolar respetuosa de las individualidades $\mathrm{y}$, por tanto, que valora la diversidad. Sin embargo, estas declaraciones no concuerdan con lo que las entrevistadas reconocen o significan conceptualmente. Prevalece un desconocimiento en lo que respecta a la educación inclusiva por parte de las agentes educativas, puesto que consideran la integración como sinónimo de inclusión, ya que las mismas declaran desarrollar prácticas inclusivas, pero al mismo tiempo utilizan conceptos inapropiados para referirse a las personas con discapacidad. En este sentido, la identificación de esta incoherencia debe provocar un proceso de capacitación que se oriente a la resocialización de las agentes en estos temas, pues queda claro que no es suficiente la transferencia de conocimiento teórico-conceptual, legislativo y procedimental respecto a la educación inclusiva para que esta pueda ser 
implementada. Se debe impactar las resistencias, mitos y aprendizajes previos que sustentan esta dicotomía.

Respecto a aspectos infraestructurales, se halla que las instituciones de los jardines infantiles han realizado algunos ajustes razonables de la Ley 20.422 (2010). No obstante, aún es posible evidenciar que la mayoría de los contextos educativos no cuentan con una infraestructura inclusiva debido a la antigüedad de su construcción, disminuyendo el acceso de matrícula de párvulos. Si bien, esta situación es subsanada a través de la gestión de matrícula en otro establecimiento de la red, la inexistencia de un fondo o programa que incentive la realización de las adaptaciones o transformaciones en la infraestructura, excluye a niños y niñas que lo requieren. Se destaca también que las escasas capacitaciones en materia de discapacidad perjudican no solo a las agentes educativas en sus quehaceres profesionales, sino que también damnifican a cada niño y niña con discapacidad. Considerando la importancia de las herramientas y materiales didácticos mencionados por las bases curriculares de la educación parvularia, se acentúa que la escasa cantidad de juegos didácticos crea conflictos en el aula. Lo anterior conduce a entender que las opciones o la oferta de juegos son limitadas, por lo que se infiere que esta situación obstaculiza lo planteado por el Ministerio de Educación de Chile (2018) donde menciona que la elección de medios, materiales y juego didácticos ayudan en el aprendizaje y en la fomentación de la autonomía en niños y niñas.

Se resalta como facilitador del proceso de inclusión, la estrecha y virtuosa relación entre las agentes educativas y las familias, puesto que tienen la libertad de participar en procesos de aprendizajes. En este sentido, la relación directa que se establece permite un acompañamiento constante a niños y niñas, y con ello, conocer sus diversidades, valorarlas e incorporarlas al proceso de enseñanza-aprendizaje

Como proyección, se considera necesario efectuar una investigación que aborde como sujetos de estudio a las familias de los niños y niñas en situación de discapacidad incorporando su visión sobre los procesos inclusivos en educación parvularia. De esto se desprende la siguiente pregunta investigativa ¿Cómo visualizan las familias de niños y niñas con discapacidad los procesos inclusivos en educación parvularia?

Las limitaciones estudio fue el número acotado de la muestra, puesto que incorporar una muestra más amplia permitiría analizar otras especificidades, relaciones y diferencias entre jardines de las instituciones investigadas. Además, complementar el desarrollo de las entrevistas semiestructuradas con observaciones participantes accedería desarrollar un 
análisis más integral en relación a las metodologías inclusivas, significados, facilitadores y obstaculizadores de los jardines infantiles de la comuna de Copiapó de la Tercera región, Chile.

\section{Referencias}

Agencia de Calidad de la Educación. (2015). Estudio de calidad educativa en educación parvularia: Experiencias internacionales y representaciones sociales nacionales. Recuperado de https://bit.ly/3jiyyKx

Cáceres, Pablo. (2003). Análisis cualitativo de contenido: una alternativa metodológica alcanzable. Psicoperspectivas, 2, 53-82. Recuperado de https://bit.ly/2ANEMSs

Calvillo Racanac, Rosa Hermelinda. (2013). Rincones de aprendizaje y desarrollo de la creatividad del niño (Tesis de Licenciatura). Universidad Rafael Landívar. Recuperado de https://bit.ly/30WGYlo

Castillo Armijo, Pablo y Miranda Carvajal, Carlos. (2018). Actitud hacia la Inclusión de los Estudiantes de Pedagogía de una Universidad Estatal Chilena. Revista latinoamericana de educación inclusiva, 12(2), 133-148. doi: https://dx.doi.org/10.4067/S0718-73782018000200133

Corrales Huenul, Angélica, Soto Hernández, Valentina y Villafañe Hormazábal, Gabriela. (2016). Barreras de aprendizaje para estudiantes con discapacidad en una universidad chilena. Demandas estudiantiles- desafíos institucionales. Revista Actualidades Investigativas en Educación, 16(3), 1-29. doi: https://doi.org/10.15517/aie.v16i3.25957

Decreto 201. (2008). Promulga la Convención de las Naciones Unidas sobre los Derechos de las personas con discapacidad y su protocolo facultativo. Recuperado de https://bit.ly/3kX3e5T

Decreto 830. (1990). Promulga convención sobre los derechos del Niño. Recuperado de https://bit.ly/3odi1em

Decreto Exento 83. (2015). Aprueba criterios y orientaciones de adecuación curricular para estudiantes con necesidades educativas especiales de educación parvularia y educación básica. Recuperado de https://bit.ly/3kEFmDI

Elige Educar. (2019). Educación Parvularia en Chile: Estado del arte y desafíos. Una propuesta de Elige Educar. Santiago, Chile: Elige Educar. Recuperado de https://bit.ly/3oc5TdG

Escobar, Nancy. (2011). La Mediación del Aprendizaje en la Escuela. Acción Pedagógica, (20), 58-73. Recuperado de https://bit.ly/3hrHDQz

Espejo, Roberto y Sarmiento, Rafael, (2017). Manual de apoyo docente: Metodologías activas para el aprendizaje. Santiago, Chile: Universidad Central de Chile. Recuperado de https://bit.ly/2RRMwaS 
Fondo de Investigación y Desarrollo en Educación. (2018). Informe final. Rol del juego en la educación paruvularia: Creencias y prácticas de educadoras del nivel transición menor. Recuperado de https://bit.ly/3jOxjTA

Grande Fariñas, Patricia y González Noriega, María del Mar. (2015). La educación inclusiva en la educación infantil: propuestas basadas en la evidencia. Tendencias pedagógicas, (26), 145-162. Recuperado de https://bit.ly/35YQGGo

Gutierrez, Mureen Jennifer. (2007). Contextos y barreras para la inclusión educativa. Horizontes Pedagógicos, 9(1), 47-56. Recuperado de https://bit.ly/3hHYyPJ

Junta Nacional de Jardines Infantiles. (2017). Informe final: Estudio sobre factores Asociados a la Permanencia de los Párvulos en Programas Educativos de la JUNJI. Santiago, Chile: JUNJI; Galerna Consultores. Recuperado de https://bit.ly/2N9fVuP

Leal Riquelme, Rubén y Herrera Guerrero, Berta. (2009). La constitución de significado en el ámbito de las relaciones intersubjetivas: El acto personal y la acción social. Alpha, (28), 135-151. https://dx.doi.org/10.4067/S0718-22012009000100009

Ley 20.422. (2010). Establece normas sobre igualdad de oportunidades e inclusión social de personas con discapacidad. Recuperado de https://bit.ly/34JWsdG

López Aguilar, Norma Graciela y Sánchez Dorantes, Ludivina. (2010). El aburrimiento en clases. Procesos Psicológicos y Sociales, 6(1-2), 1-43. Recuperado de https://bit.ly/2CobHO5

Ministerio de Educación de Chile. (2015). Diversificación de la enseñanza: Decreto N83/2015. Santiago, Chile: Ministerio de Educación de Chile. Recuperado de https://bit.ly/3kGnjwl

Ministerio de Educación de Chile. (2018). Bases Curriculares Educación Parvularia. Santiago, Chile: Ministerio de Educación de Chile. Recuperado de https://bit.ly/2YMdhAF

Mora Nawrath, Héctor. (2009). Mundo de la vida, comprensión y acción intersubjetiva en la sociología fenomenológica de Alfred Schütz. Revista CUHSO, 18(1). doi: https://doi.org/10.7770/CUHSO-V18N1-ART300

Morris, Rebecca. (1994) Computerized content analysis in management research: a demonstration of advantages \& limitations. Journal of management, 20 (4), 903-931. doi: https://doi.org/10.1016/0149-2063(94)90035-3

Muñoz Mella, Fanny Isabel. (2018). Educación Inclusiva: una mirada a las estrategias desarrolladas por las Técnicos en Educación Parvularia de un Jardín Infantil. Polyphōnía. Educación Inclusiva, 2(1), 75-97. Recuperado de https://bit.ly/3hGBIls

Niño Quiroz, Blanca Cecilia. (2016). Estrategias inclusivas en el aula. Propuesta frente a las dificultades cognitivas en el ciclo dos de educación básica en el colegio Brazuelos I.ED. (Tesis de Maestría). Universidad Distrital Francisco Jose de Caldas, Colombia. Recuperado de https://bit.ly/3ePSTo7 
Organización Mundial de la Salud. (2013). El desarrollo del niño en la primera infancia y la discapacidad: Un documento de debate. Malta: Organización Mundial de la Salud. Recuperado de https://uni.cf/2YKFTug

Organización Mundial de la Salud. (2011). Resumen sobre el Informe mundial sobre la discapacidad. Malta: Organización Mundial de la Salud. Recuperado de https://bit.ly/30YPWyn

Pedrero Lorente, Agustín. (2012). Metodología de Rincones Tradición e innovación en Eduación Infantil (3-6 años). Editorial de la Infancia, S.L.

Resolución N² 217. (1948). Declaración Universal de los Derechos Humanos. Recuperado de https://www.leychile.cl/Navegar?idNorma=1000396

Ricoy Lorenzo, Carmen. (2006). Contribución sobre los paradigmas de investigación. Educação, 31(1), 11-22. Recuperado de https://bit.ly/39kRJ2P

Rizo García, Marta. (2015). Construcción de la realidad, comunicación y vida cotidiana. Una aproximación a la obra de Thomas Luckmann. Intercom: Revista Brasileira de Ciências da Comunicação, 38(2). doi: https://doi.org/10.1590/1809-5844201522

Sánchez-Migallón, Laura Dorado. (2019). Los rincones: Contextos potencialmente significativos de aprendizaje para la inclusión (Tesis de Maestría). Universidad Complutense de Madrid. Recuperado de https://bit.ly/2YGaWXO

Sección de Participación, Género e Inclusión. (2017). Recomendaciones. Uso del lenguaje inclusivo persona en situación de discapacidad. Recuperado de https://bit.ly/2G8IM2H

Sirkko, Riikka, Kyrönlampi, Taina y Puroila, Anna-Maija. (2019). Children's Agency: Opportunities and Constraints. International Journal of Early Childhood, 51, 283-300. doi: https://doi.org/10.1007/s13158-019-00252-5

Strauss, Anselm y Corbin, Juliet. (2002). Basics of qualitative research. Techniques and procedures for developing grounded theory $\left(4^{\text {th }}\right.$ ed.). Sage Publications.

Subsecretaría de Educación Parvularia de Chile. (2018). Planificación y Evaluación. Orientaciones Pedagógicas para el nivel de Educación Parvularia. Santiago, Chile: Subsecretaría de Educación Parvularia de Chile. Recuperado de https://bit.ly/3fufzey

Superintendencia de Educación de Chile. (2017). Particularidades de la Educación Parvularia. Santiago. Recuperado de https://bit.ly/3fBJotx

Velarde Lizama, Valentina. (2012). Los modelos de la discapacidad: un recorrido histórico. Empresa \& humanismo, 15(1). 115-136. Recuperado de https://bit.ly/3dbCKsA 
Revista indizada en

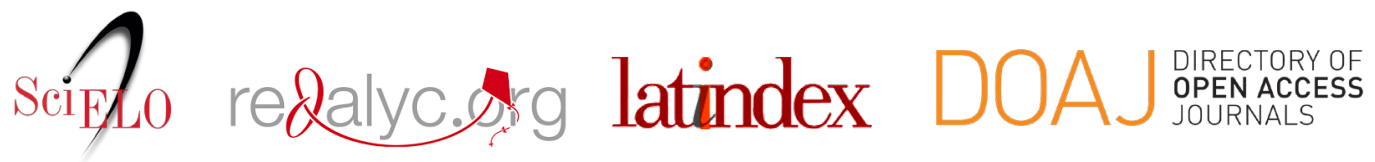

Distribuida en las bases de datos:

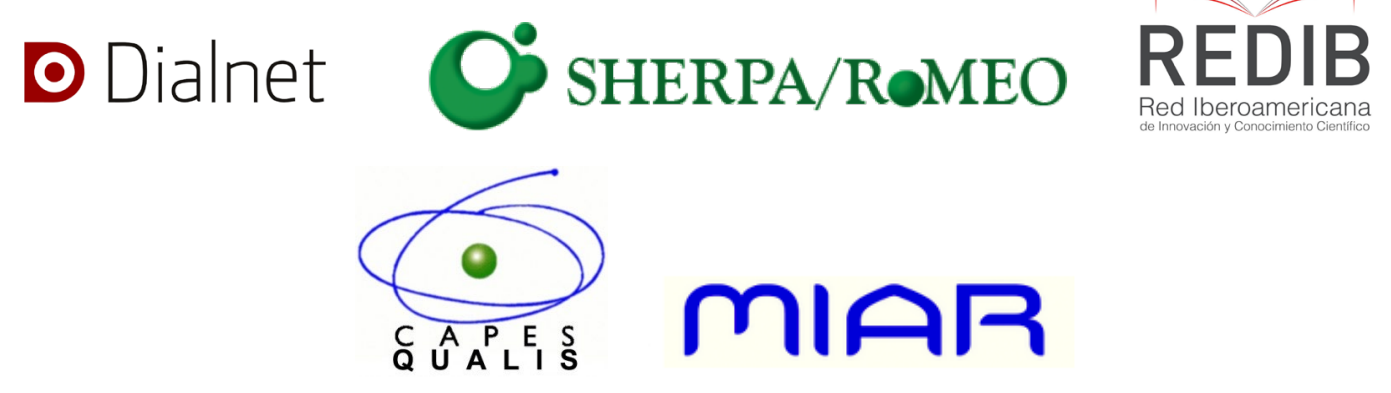

